\title{
Avaliação do efeito tóxico de Leucaena leucocephala (Leg. Mimosoideae) em ovinos ${ }^{1}$
}

\author{
Ana Paula M.G. Almeida ${ }^{2}$, Glaucia D. Kommers ${ }^{3}$, Ana Paula A. Nogueira ${ }^{4}$, Luis G.B. \\ Júnior $^{4}$, Brenda M.F. Prado Marques ${ }^{5}$ e Ricardo A.A. Lemos ${ }^{6}$

\begin{abstract}
Almeida A.P.M.G., Kommers G.D., Nogueira A.P.A., Júnior L.G.B., Marques B.M.F.P. \& Lemos R.A.A. 2006. [Evaluation of the toxicity of Leucaena leucocephala (Leg. Mimosoideae) in sheep.] Avaliação do efeito tóxico de Leucaena leucocephala (Leg.Mimosoideae) em ovinos. Pesquisa Veterinária Brasileira 26(3):190-194. Departamento de Medicina Veterinária, Faculdade de Medicina Vetetinária e Zootecnia, Universidade Federal de Mato Grosso do Sul, Av. Senador Filinto Müller 2443, Cx.Postal 549, Campo Grande, MS 79070-900, Brazil. E-mail: eqrural@nin.ufms.br

An experiment was performed to evaluate the toxicity of Leucaena leucocephala for sheep. Two ewes and six lambs were divided into seven treatments and fed the plant. The adult ewes did not show clinical signs of poisoning. The 4 to 5 -month-old lambs presented partial wool loss 6 days after beginning of the administration of the plant and total wool loss after 12 or 20 days. The age of the sheep in this experiment suggests resistance of adult ewes and susceptibility of young lambs to L. leucocaephala poisoning.
\end{abstract}

INDEX TERMS: Poisonous plants, Leucaena leucocephala, Leguminosae Mimosoideae, plant poisoning, sheep diseases, pathology.

RESUMO.- Foi desenvolvido um experimento com o objetivo de avaliar o efeito tóxico da planta Leucaena leucocephala para ovinos. A planta foi administrada a duas ovelhas adultas e seis cordeiros divididos em sete tratamentos. Os cordeiros de 4-5 meses apresentaram queda parcial de lã 6 dias após o início da administração da planta, e queda total de lã depois de 12 ou 20 dias do início da administração. As ovelhas adultas não adoeceram o que sugere certa resistência dos adultos à intoxicação por $L$. leucocephala.

TERMOS DE INDEXAÇ̃̃O: Plantas tóxicas, Leucaena leucocephala, Leguminosae Mimosoideae, intoxicação por planta, doenças de ovinos, patologia.

\footnotetext{
${ }^{1}$ Recebido em 8 de fevereiro de 2006.

Aceito para publicação em 10 de março de 2006 .

Parte da tese de mestrado do primeiro autor.

2 Aluna do Programa de Mestrado em Ciência Animal, Universidade Federal do Mato Grosso do Sul (UFMS).

${ }^{3}$ Depto Patologia, Universidade Federal de Santa Maria (UFSM), 97105900 Santa Maria, RS.

${ }^{4}$ Bolsista de iniciação científica, UFMS.

${ }^{5}$ Bolsista PIBIC/UFSM.

${ }^{6}$ Depto Medicina Veterinária, Faculdade de Medicina Vetetinária e Zootecnia, UFMS, Av. Senador Filinto Muller 2443, Cx.Postal 549, Campo Grande, MS 79050-900, Brazil. *Autor para correspondência: eqrural@nin.ufms.br
}

\section{INTRODUÇÃO}

Leucaena leucocephala é um arbusto ou árvore da família Leguminosae, sub-família Mimosoideae, originária da América Central, que atualmente encontra-se difundida pelas regiões tropicais do mundo (Tokarnia et al. 2000).

É uma planta altamente nutritiva, palatável e de boa digestibilidade, mas sua utilização como forrageira tem sido limitada por seu teor em mimosina, a qual possui efeitos tóxicos caracterizados por alopecia e menos freqüientemente, catarata, atrofia de gengiva, ulcerações da língua e esôfago, bócio, infertilidade e menores ganho de peso (Radostits et al. 2000) para ruminantes e não-ruminantes (Hammond 1995, Radostits et al. 2000, Tokarnia et al. 2000).

O grande potencial para o desenvolvimento da ovinocultura de corte em Mato Grosso do Sul (Carneiro 2002) e a possibilidade da utilização de L. leucocephala como importante forrageira em nosso meio (Tokarnia et al. 2000), justificam o presente trabalho, que tem por objetivo avaliar o efeito tóxico de L. leucocephala para ovinos.

\section{MATERIAL E MÉTODOS}

A Leucaena leucocephala (Lam.) de Wit. foi administrada a 8 ovinos em 7 tratamentos diferentes, conforme descrito no Quadro 1.

Os ovinos ficaram alojados em baias individuais de alvenaria com área de $4 \mathrm{~m}^{2}$. A planta foi fornecida na forma de folhas verdes recémcortadas, servidas no comedouro 2 vezes ao dia. A quantidade de planta diária fornecida foi pesada e caso ocorressem sobras, o excesso 
Quadro 1. Distribuição dos tratamentos, número e categoria de animais e composição da dieta do experimento para determinação da toxicidade de Leucaena leucocephala para ovinos

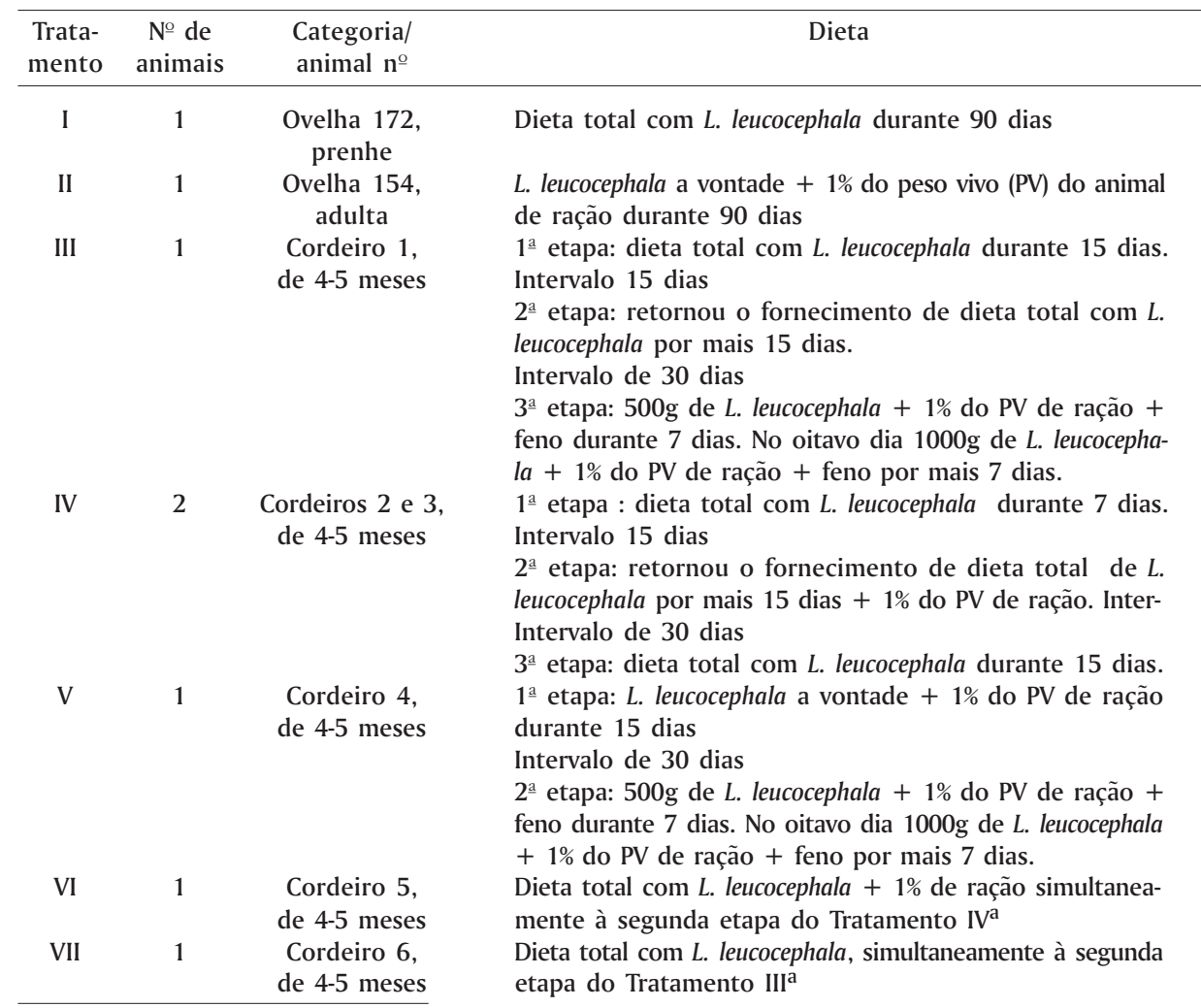

${ }^{\text {a }}$ Com o objetivo de avaliar se a planta mantém a sua toxicidade.

era pesado para estimativa da quantidade de planta consumida. Os animais consumiam $2 \mathrm{~kg}$ da planta/dia. $\mathrm{O}$ fornecimento de água foi à vontade. Durante os períodos de suspensão de L. leucocephala, os ovinos foram alimentados com feno de tifton e $2 \%$ peso vivo de ração comercial formulada para ovinos. Antes do início do experimento, os ovinos foram pesados individualmente, vermifugados, e suas fezes colhidas para realização de exames parasitológicos pela técnica de ovos por grama de fezes (OPG). Esses procedimentos foram repetidos com intervalos de 10 dias. Identificaram-se todos os ovinos por códigos através de brincos numerados (Ovelhas 154 e 172, Cordeiros 1, 2, 3, 4, 5 e 6) e os dados de cada animal foram registrados em fichas individuais.

Realizaram-se biópsias de pele de $1 \mathrm{~cm}^{2} \mathrm{em}$ todos os ovinos antes do início da administração da planta, e posteriormente em intervalos de 7 dias. 0 material para biópsia foi colhido na região lombar e a distância entre as biópsias foi de $5 \mathrm{~cm}$ no sentido caudal. A técnica da biópsia consistiu de depilação, desinfecção do local com álcool iodado, anestesia local com lidocaína a $2 \%$ e coleta da amostra de pele com bisturi. Os ferimentos foram tratados com cicatrizantes e repelentes. Os fragmentos de pele foram fixados em formol neutro a $10 \%$ e processados para histopatologia. Os animais foram submetidos diariamente à avaliação dos seguintes parâmetros clínicos: frequiências cardíaca e respiratória, coloração de mucosas, temperatura e resistência à tração manual da lã.

Esse experimento foi protocolado sob o no.95/2005 e aprovado pela Comissão de Ética no Uso de Animais, da Universidade Federal de Mato Grosso do Sul.

\section{RESULTADOS}

A Ovelha 172 do Tratamento I não apresentou sinais clínicos da intoxicação e pariu um cordeiro macho normal com $2,8 \mathrm{~kg}$, após 35 dias do início do experimento. A Ovelha 154 do Tratamento II também não apresentou sinais clínicos.

O Cordeiro 1 do Tratamento III apresentou desprendimento de lã após 6 dias do início da administração da planta (Fig.1), e queda total da lã após 20 dias do início da administração (Fig.2). Na segunda etapa, após 15 dias de intervalo, este animal apresentou desprendimento de lã após 6 dias do reinício da administração da planta e queda total de lã após 12 dias de consumo da

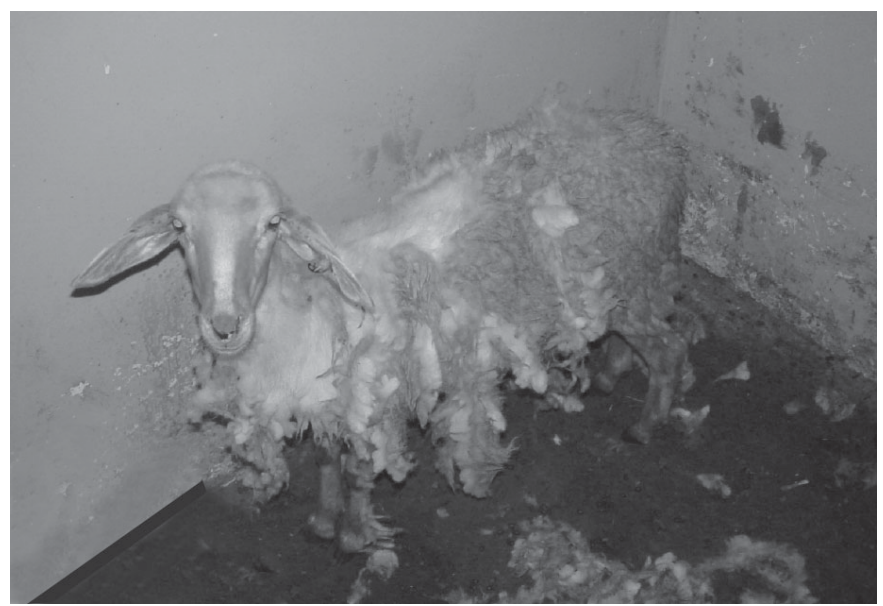

Fig.1. Cordeiro 1 (Tratamento III), apresentando desprendimento espontâneo da lã 6 dias após dieta total com Leucaena leucocephala. 


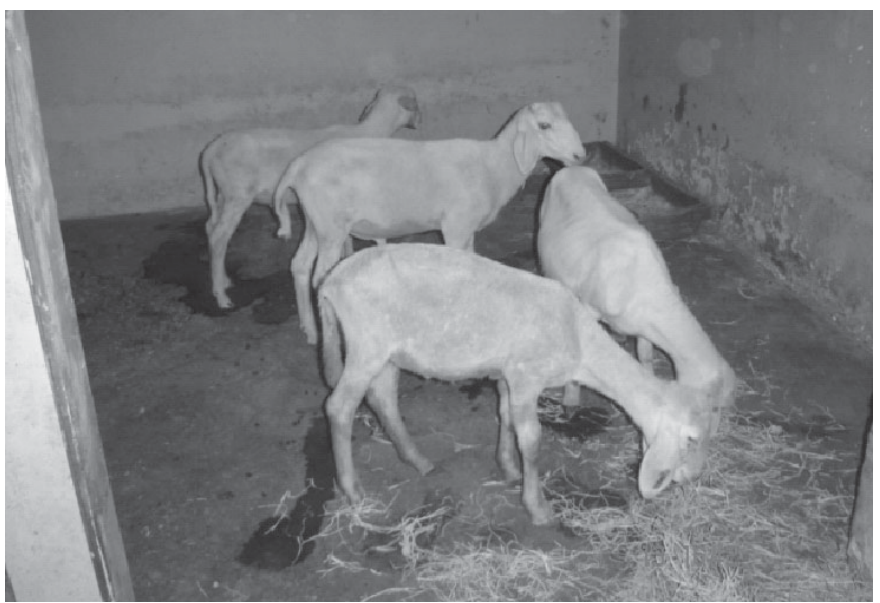

Fig.2. Cordeiros 1, 2, 3, e 6 apresentando queda total da lã após 23 dias do início da administração de Leucaena leucocephala.

mesma. Na terceira etapa, após 30 dias de intervalo, este animal não apresentou sinais clínicos da intoxicação. Os Cordeiros 2 e 3 do Tratamento IV apresentaram desprendimento de lã após 6 dias de administração da planta e queda total de lã após 20 dias (Fig.2) de consumo de L. leucocephala. Na segunda etapa, após 15 dias de intervalo, ambos apresentaram desprendimento de lã 9 dias após o reinício da administração da planta. O Cordeiro 2 apresentou queda total da lã após 17 dias do início da administração da planta e o Cordeiro 3 recuperou-se após a suspensão do fornecimento da planta. Na terceira etapa, após 30 dias de intervalo, ambos apresentaram discreto desprendimento de lã 6 dias após o início da administração da planta e recuperaram-se após a suspensão.

O Cordeiro 4 do Tratamento $\mathrm{V}$ apresentou desprendimento de lã após 12 dias do início da administração da planta e recuperou-se após a suspensão da mesma. Após o intervalo de 30 dias, este animal não apresentou sinais clínicos da intoxicação.

O Cordeiro 5 do Tratamento VI apresentou desprendimento de lã após 9 dias do início da administração da planta e recuperou-se após a suspensão. O Cordeiro 6 do Tratamento VII apresentou desprendimento da lã após 9 dias do início da administração da planta e queda total da lã (Fig.2), redução de apetite e lacrimejamento, após 2 dias da suspensão da mesma, e recuperou-se após 17 dias da suspensão.

Não foram observadas alterações nos parâmetros clínicos avaliados e nos exames parasitológicos realizados. Todos os tratamentos conferiram ganho de peso aos animais, exceto o Tratamento VII (Quadro 2).

Quadro 2. Diferença de peso ao final do experimento para determinação da toxicidade da Leucaena leucocephala para ovinos

\begin{tabular}{ccccc}
\hline Tratamento & $\begin{array}{c}\text { Animal } \\
\mathbf{n}{ }^{\circ}\end{array}$ & $\begin{array}{c}\text { Peso inicial } \\
(\mathrm{kg})\end{array}$ & $\begin{array}{c}\text { Peso final } \\
(\mathrm{kg})\end{array}$ & $\begin{array}{c}\text { Ganho de peso } \\
(\mathrm{kg} / \mathrm{dias})\end{array}$ \\
\hline I & 172 & 34 & 38 & $4 \mathrm{~kg} / 90 \mathrm{dias}$ \\
II & 154 & 32 & 40 & $8 \mathrm{~kg} / 90 \mathrm{dias}$ \\
III & 1 & 17 & 25 & $8 \mathrm{~kg} / 90 \mathrm{dias}$ \\
IV & 2 & 18 & 23 & $5 \mathrm{~kg} / 82 \mathrm{dias}$ \\
& 3 & 18 & 22 & $4 \mathrm{~kg} / 82 \mathrm{dias}$ \\
V & 4 & 16 & 23 & $7 \mathrm{~kg} / 60 \mathrm{dias}$ \\
VI & 5 & 16 & 18 & $2 \mathrm{~kg} / 15 \mathrm{dias}$ \\
VII & 6 & 16 & 16 & $0 \mathrm{~kg} / 15 \mathrm{dias}$
\end{tabular}

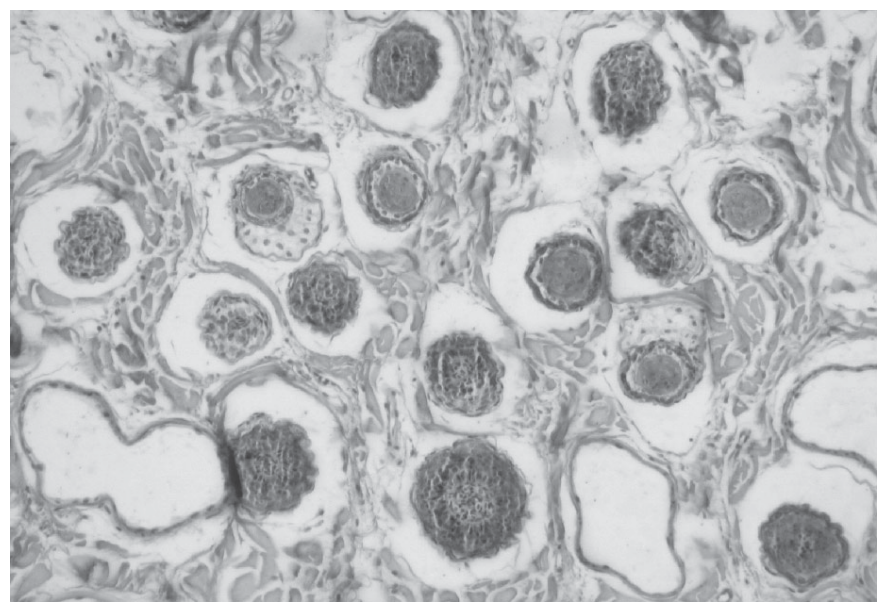

Fig.3. Histologia da biópsia de pele do Cordeiro 6, realizada 15 dias após o início da administração de Leucaena leucocephala como alimento exclusivo. A grande maioria dos folículos encontra-se em fase telógena, com marcada redução no tamanho dos folículos e fechamento da luz folicular.

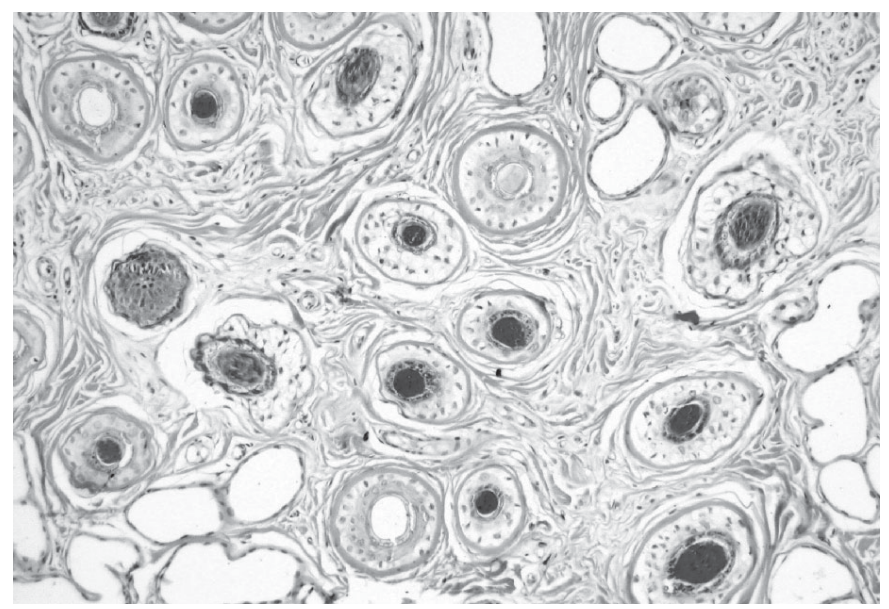

Fig.4. Histologia da biópsia de pele da Ovelha 172 , realizada no animal antes do início do recebimento de Leucaena leucocephala como alimento exclusivo. A grande maioria dos folículos encontra-se em fase anágena.

Nas biópsias de pele, alterações relacionadas aos folículos da lã foram observadas nos Cordeiros 1 (Tratamento III), 2 (Tratamento IV) e 6 (Tratamento VII).

No Cordeiro 1 (Tratamento III) observou-se o contorno levemente pregueado da bainha radicular externa (BRE) de alguns folículos em anágeno (fase de crescimento). Este cordeiro apresentou desprendimento parcial da lã após 6 dias de consumo da planta na segunda etapa do experimento.

No Cordeiro 2 (Tratamento IV), a maioria dos folículos estavam diminuídos de tamanho e com pregueamento da BRE, encontrando-se muitos deles em fase telógena (fase de repouso) e outros em fase anágena (com haste de lã na luz folicular). Este ovino consumiu exclusivamente a planta por 7 dias, após período de recuperação de 15 dias, consumiu a planta associada a 1\% do PV de ração e apresentou desprendimento total da lã. 
Na pele do Cordeiro 6 (Tratamento VII), o qual teve desprendimento de lã após 9 dias de consumo exclusivo de L. leucocephala, observou-se a grande maioria dos folículos em fase de crescimento (fase anágena), porém alguns folículos tinham contorno irregular (pregueado) da BRE. Aos 15 dias de consumo exclusivo da planta, a grande maioria dos folículos estava em fase de repouso (fase telógena), caracterizada por marcada redução no tamanho dos folículos, com fechamento da luz folicular. Observou-se também o pregueamento do contorno externo da BRE dos folículos na fase telógena e também de alguns folículos remanescentes em fase anágena, os quais ainda continham hastes de lã na luz folicular (Fig.3).

$O$ resultado padrão das biópsias que foram realizadas nos ovinos no período de adaptação, antes de consumirem $L$. leucocephala, foi a observação da grande maioria dos folículos em fase anágena (Fig.4).

\section{DISCUSSÃO E CONCLUSÕES}

Leucaena leucocephala demonstrou ser experimentalmente tóxica para ovinos de 4-5 meses de idade. O principal sinal clínico observado foi perda parcial ou total de lã. Apenas um animal apresentou anorexia, emagrecimento e lacrimejamento. Esses sinais estão de acordo com outros relatos (Hammond 1995, Radostits et al. 2000, Tokarnia et al. 2000, Riet-Correa et al. 2004).

O início dos sinais clínicos ocorreu 6-9 dias após o início da administração exclusiva da planta, e 9-12 dias após o início da administração da planta associada a $1 \%$ de ração. Estes dados estão de acordo com o período descrito por Radostits et al (2000). Outros autores descrevem o início dos sinais clínicos com 10 (Franzolin Neto \& Veloso 1986) ou 15 dias após o início da administração da planta (Riet-Correa et al. 2004).

Todos os ovinos com idade de 4-5 meses apresentaram queda de lã, o que não foi observado nas ovelhas adultas. Na literatura consultada não foram encontradas citações sobre a influência da idade no aparecimento dos sinais clínicos.

No presente estudo, a reintrodução de L. leucocephala na alimentação dos cordeiros que já haviam apresentado sinais clínicos, a intoxicação numa primeira fase, após intervalo de 15 dias, provocou novamente $o$ aparecimento de sinais clínicos mais rapidamente do que naqueles que não haviam ingerido a planta previamente. É possível que ainda houvesse atividade residual do princípio tóxico no organismo, potencializando essa precocidade no aparecimento dos sinais clínicos.

Porém numa terceira etapa de alimentação com L. leucocephala, após um intervalo de 30 dias, os animais não mostraram sinais clínicos ou só discreto desprendimento da lã, o que sugere uma possível adaptação da flora ruminal com degradação da mimosina após intervalos maiores. Deve-se também, considerar que o avanço da idade dos cordeiros pode ter influenciado na ausência ou no aparecimento de sinais clínicos mais brandos na terceira etapa do experimento.

Franzolin Neto \& Veloso (1986) verificaram acentuada queda dos pêlos no $10^{\circ}$ dia, e queda total de lã 4 dias depois, em nove de um total de dez ovinos que receberam feno de $L$. leucocephala como alimento exclusivo; esses dados assemelham-se aos resultados obtidos nos Tratamentos III e IV.
Nos experimentos de Carvalho \& Languidey (1992), de cinco ovinos que receberam as folhas frescas de L. leucocephala como alimento exclusivo, um animal apresentou anorexia, alopecia, perda de peso, sialorréia e incoordenação motora, semelhantemente aos sinais clínicos do Cordeiro 6 do Tratamento VII, que não apresentou sialorréia e incoordenação motora, mas apresentou lacrimejamento. Em ambos os experimentos os animais recuperaram-se após a suspensão da administração da planta.

Riet-Correa et al. (2004) observaram que, dois de quatro ovinos que receberam $L$. leucocephala experimentalmente, apresentaram severa salivação 15 dias após o início da administração, o que não foi observado em nenhum grupo deste experimento. Sinais severos da intoxicação tais como catarata, atrofia de gengiva, ulcerações da língua e esôfago, bócio, infertilidade e menor ganho de peso não foram observados neste experimento. Um ovino, relatado por Riet-Correa et al. (2004), apresentou duas áreas de alopecia de $2 \mathrm{~cm}$ de diâmetro na pele do pescoço e região escapular esquerda, semelhante aos sinais clínicos observados nos Tratamentos V, IV ( $3^{\circ}$ etapa) e VI. Após a suspensão da administração da planta um ovino recuperou-se, como os animais dos Tratamentos $\mathrm{V}$, IV ( $3^{\circ}$ etapa) e VI, e o outro continuou salivando, com anorexia e perda de peso progressiva, morrendo 11 dias após o final do consumo. No presente estudo, todos os animais recuperaram-se após a suspensão da administração da planta.

A acentuada telogenização (folículos em repouso) folicular, observada nas biópsias cutâneas de dois cordeiros deste experimento, pode estar relacionada aos efeitos tóxicos da mimosina. Quando infusões venosas deste princípio tóxico foram administradas a ovinos, as alterações de pele consistiam de retrocesso folicular e de uma parada abrupta e temporária no crescimento da lã, sem efeitos tóxicos colaterais, permitindo a remoção manual da lã (Reis et al. 1975, Reis \& Panaretto 1979). O efeito depilatório agudo da leucena em ruminantes possivelmente se deve a ação da mimosina incompletamente degradada no rúmen (Tokarnia et al. 2000).

Nas condições experimentais deste trabalho concluiu-se que os Cordeiros de 4-5 meses mostraram-se sensíveis à toxicidade de $L$. leucocephala, apresentando acentuada queda de lã por volta de 6 dias após o início do consumo da planta, porém ganharam peso no decorrer do experimento. As ovelhas adultas não apresentaram sinais clínicos da intoxicação e também ganharam peso no decorrer do experimento. A idade dos ovinos neste experimento sugere certa resistência dos adultos e susceptibilidade dos jovens à intoxicação por $L$. leucocephala. A adição de $1 \%$ de ração na dieta não interferiu nos efeitos tóxicos da planta em cordeiros de 4-5 meses.

Para a plena utilização de $L$. leucocephala na alimentação animal, há necessidade de um maior conhecimento do seu potencial tóxico e das condições em que ocorre a intoxicação, como evidenciado nos ovinos de alguns tratamentos deste estudo, visando principalmente encontrar um sistema eficiente de detoxificação microbiana ruminal que permita o consumo da planta sem a ocorrência de intoxicação.

Agradecimentos.- À EQUALI (Escola de Qualificação Rural) da FMVZ/UFMS pela compra dos ovinos utilizados no experimento. 


\section{REFERÊNCIAS}

Carneiro L.O. 2002. A ovinocultura de corte no Mato Grosso do Sul: uma alternativa econômica. Tese especialização MBA ??? Gestão Estratégica de Agronegócios. Departamento de Economia e Administração, UFMS, Campo Grande, MS..

Carvalho O.M. \& Languidey P.H. 1992. Toxidez de Leucaena leucocephala em ovinos Sta Inês. Revta Soc. Bras. Zootec. 21(1):1-9.

Franzolin Neto R. \& Velloso L. 1986. Leucaena leucocephala (Lam.) de Wit em rações para ovinos. Toxicidade. Revta Soc. Bras. Zootec. 15(5):415-424.

Hammond A.C. 1995. Leucaena toxicosis and its control in ruminants. J. Anim. Sci. 73:1487-1492.

Jones R.J. 1985. Leucaena toxicity and the ruminal degradation of mimosine, p.111. In: Seawright A.A., Hegarty M.P., James L.F. \& Keeler R.F. (ed.) Plant Toxicology. Queensland Poisonous Plants Committee, Yeerongpilly, Queensland, Australia.
Luo J., Litherland A.J., Sahlu T., Puchala R., Lachica M. \& Goetsch A.L. 2000. Effects of mimosine on fiber shedding, follicle activity, and fiber regrowth in Spanish goats. J. Anim. Sci. 78:1551-1555.

Radostits O.M., Blood D.C. \& Gay C.C. 2000. Veterinary Medicine. 8th ed. Bailliere Tindal, London. 1773p.

Reis P.J., Tunks D.A. \& Chapman R.E. 1975. Effects of mimosine, a potential chemical defleecing agent, on wool growth and the skin of sheep. Aust. J. Biol. Sci. 28:69-84. (Cit. Luo et al. 2000)

Reis P.J. \& Panaretto B.A. 1979. Chemical defleecing as a method of harvesting wool from sheep. World Anim. Rev. 30:36-42. (Cit. Luo et al. 2000)

Riet-Correa F., Vilar T.F.M., Araújo J.A.S., Santos J.C.A. \& Medeiros R.M. 2004. Intoxicação por Leucaena leucocephala em ovinos na Paraíba. Pes. Vet. Bras. 24(Supl.):52.

Tokarnia C.H, Döbereiner J. \& Peixoto P.V. 2000. Plantas Tóxicas do Brasil. Editora Helianthus, Rio de Janeiro. 310p. 